\title{
BRST approach to Lagrangian formulation of bosonic totally antisymmeric tensor fields in curved space
}

\author{
I.L. BuChBindeR ${ }^{a *}$, V.A. KRYKhtin ${ }^{a, b \dagger}$, L.L. RYskinA ${ }^{a \ddagger}$ \\ ${ }^{a}$ Department of Theoretical Physics, \\ Tomsk State Pedagogical University, \\ Tomsk 634061, Russia \\ ${ }^{b}$ Laboratory of Mathematical Physics, \\ Tomsk Polytechnic University, \\ Tomsk 634050, Russia
}

\begin{abstract}
We apply the BRST approach, previously developed for higher spin field theories, to gauge invariant Lagrangian construction for antisymmetric massive and massless bosonic fields in arbitrary d-dimensional curved space. The obtained theories are reducible gauge models both in massless and massive cases and the order of reducibility grows with the value of the rank of the antisymmetric field. In both the cases the Lagrangians contain the sets of auxiliary fields and possess more rich gauge symmetry in comparison with standard Lagrangian formulation for the antisymmetric fields. This serves additional demonstration of universality of the BRST approach for Lagrangian constructions in various field models.
\end{abstract}

\section{Introduction}

BRST-BFV- construction [1], which was initially developed for quantization of gauge theories, turned out to be the power method for deriving the Lagrange formulation in higher spin field theory]. Indeed, this method has successfully been applied to finding the Lagrangians of massless and massive, bosonic and fermionic higher spin fields with various symmetry structure of indices in Minkowski and AdS spaces [3-8]2. This method, named BRST-approach to higher spin field theory, in all cases yields to gauge invariant action in terms of off-shell totally unconstrained fields and gauge parameters.

The BRST-approach begins with postulating the operator constraints, determining the irreducible representation of Poincare or AdS algebra with given spin and constructing the closed algebra of these operators. It should be pointed out that closing the algebra requires to introduce some new operators, which can not be interpreting as the constraints. Also, closing the algebra imposes the restrictions

*joseph@tspu.edu.ru

†krykhtin@tspu.edu.ru

${ }_{\ddagger}^{\ddagger}$ ryskina@tspu.edu.ru

${ }^{1}$ Aspects of modern state of higher spin field theory are discussed in the reviews [2].

${ }^{2}$ Recently this approach was applied to Lagrangian formulation of interacting bosonic higher spin gauge fields [9]. 
on space-time geometry and it turns out to be that in general the scheme under considerations works only for constant curvature space: 3 . As we will see, there is a non-trivial case when this scheme successfully works for arbitrary d-dimensional curved space.

In this paper we develop the BRST-approach to constructing the Lagrangian formulation for massless and massive totally antisymmetric bosonic fields in arbitrary curved spacel. We show that the general procedure, described in [3,5-8] does not impose any restrictions on space-time geometry and yields to gauge invariant model containing, besides the basic field $\varphi_{\mu_{1} \ldots \mu_{p}}$, some number of auxiliary fields. After eliminating the auxiliary fields the obtained formulation coincides with standard one.

As is well known an antisymmetric bosonic field of rank- $p \varphi_{\mu_{1} \ldots \mu_{p}}$ will realize irreducible representation of the Poincare group (in Minkowski spacetime) if the following equations are satisfied

$$
\left(\partial^{2}-m^{2}\right) \varphi_{\mu_{1} \ldots \mu_{p}}=0, \quad \partial^{\mu_{1}} \varphi_{\mu_{1} \ldots \mu_{p}}=0 .
$$

When we turn to an arbitrary curved spacetime we suppose that conditions on $\varphi_{\mu_{1} \ldots \mu_{p}}$ which must be satisfied, tend to (1) in flat space limit. It tells us that the equations on $\varphi_{\mu_{1} \ldots \mu_{p}}$ in curved spacetime must be of the form

$$
\left(\nabla^{2}-m^{2}\right) \varphi_{\mu_{1} \ldots \mu_{p}}+\text { terms with curvature }=0, \quad \nabla^{\mu_{1}} \varphi_{\mu_{1} \ldots \mu_{p}}=0,
$$

We will see that the "terms with curvature" are to be defined uniquely in process of Lagrangian construction.

The paper is organized as follows. In Section 2 we develop the BRST approach for massless antisymmetric bosonic fields. In Section 3 we consider this approach for massive antisymmetric bosonic fields. Section 4 is devoted to discussion of the results.

\section{Lagrangian construction for massless fields}

To avoid explicit manipulations with a big number of indices it is convenient to introduce the Fock space generated by fermionic creation and annihilation operators with tangent space indices

$$
\left\{a_{a}, a_{b}^{+}\right\}=\eta_{a b}, \quad \eta_{a b}=\operatorname{diag}(-,+,+, \cdots,+) .
$$

As usual the tangent space indices and the curved indices are converted one into another with the help of the vielbein $e_{\mu}^{a}$ which is assumed to satisfy the relation $\nabla_{\mu} e_{\nu}^{a}=0$. Then one introduces a derivative operator

$$
D_{\mu}=\partial_{\mu}+\omega_{\mu}^{a b} a_{a}^{+} a_{b}, \quad D_{\mu}|0\rangle=\partial_{\mu}|0\rangle=0
$$

which acts on an arbitrary state vector in this Fock space

$$
|\varphi\rangle=\sum_{p=0} \varphi_{\mu_{1} \ldots \mu_{p}}(x) a^{\mu_{1}+} \ldots a^{\mu_{p}}|0\rangle
$$

as the covariant derivative operator

$$
D_{\mu}|\varphi\rangle=\sum_{p=0}\left(\nabla_{\mu} \varphi_{\mu_{1} \ldots \mu_{p}}\right) a^{\mu_{1}+} \ldots a^{\mu_{p}}|0\rangle .
$$

\footnotetext{
${ }^{3}$ Of course in case of spins $s=0, \frac{1}{2}, 1$ the above algebra is closed for arbitrary space-time geometry.

${ }^{4}$ The totally antisymmetric fields are the partial case of arbitrary mixed symmetry higher spin fields. Aspects of Lagrangian formulation for such fields are discussed in the recent papers [10-15].

${ }^{5}$ The traceless condition, which is important for general-type higher spin fields, be trivial for antisymmetric fields. We use the metric with mostly plus signature.
} 
Now we want to realize equations (2) (with $m=0$ ) as operator constraints in the Fock space. For this purpose let us define operators

$$
l_{0}=D^{2}+X, \quad l_{1}=-i a^{\mu} D_{\mu}
$$

where $D^{2}=g^{\mu \nu}\left(D_{\mu} D_{\nu}-\Gamma_{\mu \nu}^{\sigma} D_{\sigma}\right)$ and the operator $X$ is responsible for the "terms with curvature" in the first equation of (2)). Then the equations

$$
l_{0}|\varphi\rangle=0, \quad l_{1}|\varphi\rangle=0
$$

are equivalent to the corresponding equations in (2).

In order to construct Lagrangian within the BRST approach we must have at hand a set of operators which is invariant under Hermitian conjugation and which forms an algebra [6]. We assume the standard scalar product in the Fock space and suppose that operator $X$ and hence operator $l_{0}$ are hermitian with respect to this scalar product. The operator conjugate to $l_{1}$ we denote as $l_{1}^{+}$

$$
l_{1}^{+}=-i a^{\mu+} D_{\mu} .
$$

Now set of operators $l_{0}, l_{1}, l_{1}^{+}$is invariant under Hermitian conjugation. Then we must realize the second requirement: one should obtain set of operators which form an algebra. For this purpose we find all (anti)commutators generated by $l_{0}, l_{1}, l_{1}^{+}$. Since operator $l_{0}$ is not yet defined we calculate the anticommutator $\left\{l_{1}, l_{1}^{+}\right\}$. One has

$$
\left\{l_{1}, l_{1}^{+}\right\}=-D^{2}-R_{\mu \nu \alpha \beta} a^{+\mu} a^{\nu} a^{+\alpha} a^{\beta}
$$

where $R_{\beta \mu \nu}^{\alpha}=\partial_{\mu} \Gamma_{\nu \beta}^{\alpha}-\partial_{\nu} \Gamma_{\mu \beta}^{\alpha}+\Gamma_{\mu \rho}^{\alpha} \Gamma_{\nu \beta}^{\rho}-\Gamma_{\nu \rho}^{\alpha} \Gamma_{\mu \beta}^{\rho}$. Since the rhs of (10) contains operator $D^{2}$ which are present in operator $l_{0}$ we rewrite (10) as follows

$$
\left\{l_{1}, l_{1}^{+}\right\}=-l_{0}+X-R_{\mu \nu \alpha \beta} a^{+\mu} a^{\nu} a^{+\alpha} a^{\beta} .
$$

From (11) we see that in order to close the algebra we must put $X=R_{\mu \nu \alpha \beta} a^{+\mu} a^{\nu} a^{+\alpha} a^{\beta}$ and as a consequence we have operator $l_{0}$ in the form

$$
l_{0}=D^{2}+R_{\mu \nu \alpha \beta} a^{+\mu} a^{\nu} a^{+\alpha} a^{\beta} .
$$

One can check that set of operators $l_{0}, l_{1}, l_{1}^{+}$form an algebra

$$
\left\{l_{1}, l_{1}^{+}\right\}=-l_{0}, \quad\left\{l_{1}, l_{1}\right\}=\left\{l_{1}^{+}, l_{1}^{+}\right\}=\left[l_{1}, l_{0}\right]=\left[l_{1}^{+}, l_{0}\right]=0 .
$$

Thus now we have at hand set of operators which is invariant under Hermitian conjugation and form an algebra. Let us note that found expression for operator $l_{0}$ gives the following mass-shell equation on antisymmetric field of rank- $p$ in arbitray curved space

$$
\nabla^{2} \varphi_{\mu_{1} \ldots \mu_{p}}+(-1)^{p} p R_{\left[\mu_{1}\right.}^{\alpha} \varphi_{\left.\mu_{2} \ldots \mu_{p}\right] \alpha}-p(p-1) R_{\left[\mu_{1}\right.}^{\alpha \beta}{ }_{\mu_{2}} \varphi_{\left.\mu_{3} \ldots \mu_{p}\right] \alpha \beta}=0
$$

where the square brackets denote antisymmetrization

$$
A_{\left[\alpha_{1} \ldots \alpha_{p}\right]}=\frac{1}{p !}\left[A_{\alpha_{1} \ldots \alpha_{p}} \pm(p !-1) \text { permutations }\right] .
$$

Let us turn to construction of the Lagrangians. Among the operators of the algebra (13) there are no operators which are not constraints. All the operators are constraints in the bra-vector space or/and in the ket-vector space

$$
\left\langle\varphi\left|l_{0}=\left\langle\varphi\left|l_{1}^{+}=0, \quad l_{0}\right| \varphi\right\rangle=l_{1}\right| \varphi\right\rangle=0 .
$$


Therefore for constructing Lagrangians there is no need to introduce enlarged expressions for the operators [6] and one may construct BRST operator from the operators $l_{0}, l_{1}, l_{1}^{+}$. For this we introduce ghost 'coordinates' $\eta_{0}, q_{1}^{+}, q_{1}$ and canonically conjugated them 'momenta' $\mathcal{P}_{0}, p_{1}, p_{1}^{+}$with nonvanishing (anti)commutators

$$
\left\{\eta_{0}, \mathcal{P}_{0}\right\}=1, \quad\left[q_{1}, p_{1}^{+}\right]=\left[q_{1}^{+}, p_{1}\right]=i .
$$

After this one finds the BRST operator

$$
Q=\eta_{0} l_{0}+q_{1}^{+} l_{1}+q_{1} l_{1}^{+}+q_{1}^{+} q_{1} \mathcal{P}_{0}, \quad Q^{2}=0 .
$$

Further we define the representation of the Hilbert space where the BRST operators acts as follows

$$
a^{\mu}|0\rangle=q_{1}|0\rangle=p_{1}|0\rangle=\mathcal{P}_{0}|0\rangle=0
$$

and as a consequence the general form of the state vector in the Hilbert space is

$$
|\Phi\rangle=\sum_{k_{i}} \eta_{0}^{k_{1}}\left(q_{1}^{+}\right)^{k_{2}}\left(p_{1}^{+}\right)^{k_{3}} a^{+\mu_{1}} \ldots a^{+\mu_{k_{0}}} \Phi_{\mu_{1} \ldots \mu_{k_{0}}}^{k_{1} k_{2} k_{3}}(x)|0\rangle
$$

The sum in (201) is taken over $k_{1}$ running from 0 to 1 and over $k_{2}, k_{3}, k_{0}$ running from 0 to infinity.

Let us introduce operator

$$
\sigma_{0}=a_{\mu}^{+} a^{\mu}+i q_{1}^{+} p_{1}-i p_{1}^{+} q_{1}, \quad\left[Q, \sigma_{0}\right]=0
$$

which commute with the BRST operator. This operator is used for constructing Lagrangian with given spin $p$. For this we restrict the fields $|\Phi\rangle$ and the gauge parameters $\left|\Lambda^{(i)}\right\rangle$ in the extended Fock space including ghosts (20) by the conditions

$$
\sigma_{0}|\Phi\rangle=p|\Phi\rangle, \quad \sigma_{0}\left|\Lambda^{(i)}\right\rangle=p\left|\Lambda^{(i)}\right\rangle .
$$

If we omit these conditions then the Lagrangian (and the gauge transformations) will contain fields with all spins simultaneously. One can show (see e.g. [6]) that Lagrangian can be written as

$$
\mathcal{L}=\int d \eta_{0}\langle\Phi|Q| \Phi\rangle
$$

which is invariant under the reducible gauge transformations

$$
\delta|\Phi\rangle=Q\left|\Lambda^{(0)}\right\rangle, \quad \ldots \quad \delta\left|\Lambda^{(i)}\right\rangle=Q\left|\Lambda^{(i+1)}\right\rangle, \quad \ldots \quad \delta\left|\Lambda^{(p-2)}\right\rangle=Q\left|\Lambda^{(p-1)}\right\rangle .
$$

The chain of the gauge transformation for each given spin $p$ is finite due to (22) and to the ghost number restriction

$$
g h(|\Phi\rangle)=0, \quad g h\left(\left|\Lambda^{(i)}\right\rangle=-(i+1) .\right.
$$

Thus the Lagrangian for the massless bosonic antisymmetric field in an arbitrary curved background is constructed.

Let us show that Lagrangian (23) reproduces equations of motion (2), (14) [or the same (8)] after gauge fixing. Let us fix the rank of the antisymmetric field to be $p$. In this case we have $p-1$ reducibility stages of the gauge symmetry and due to (22) and the ghost number restriction (25) the lowest stage gauge parameter $\left|\Lambda^{(p-1)}\right\rangle$ can not depend on ghost $\eta_{0}: \mathcal{P}_{0}\left|\Lambda^{(p-1)}\right\rangle=0$. Then we introduce the following decomposition on ghost $\eta_{0}$ of the gauge parameters

$$
\left|\Lambda^{(i)}\right\rangle=\left|\Lambda_{0}^{(i)}\right\rangle+\eta_{0}\left|\Lambda_{1}^{(i)}\right\rangle .
$$


One can show that using gauge transformation for the $p-2$ stage gauge parameter

$$
\delta\left|\Lambda_{0}^{(p-2)}\right\rangle=\left(q_{1}^{+} l_{1}+q_{1} l_{1}^{+}\right)\left|\Lambda_{0}^{(p-1)}\right\rangle, \quad \delta\left|\Lambda_{1}^{(p-2)}\right\rangle=l_{0}\left|\Lambda_{0}^{(p-1)}\right\rangle
$$

we can get rid of the dependence of $p-2$ stage gauge parameter on ghost $\eta_{0}$. Then we can repeat the procedure and get rid of the dependence of $p-3$ stage gauge parameter on ghost $\eta_{0}$ using remaining part $\left|\Lambda_{0}^{(p-2)}\right\rangle$ of the gauge parameter $\left|\Lambda^{(p-2)}\right\rangle$. Applying the same procedure further we remove dependence of the gauge parameter $\left|\Lambda^{(0)}\right\rangle$ on ghost $\eta_{0}$. Thus it remains only part of the gauge parameter $\left|\Lambda^{(0)}\right\rangle$ which independent of $\eta_{0}:\left|\Lambda_{0}^{(0)}\right\rangle$. Now we decompose field $|\Phi\rangle$ and gauge parameter $\left|\Lambda_{0}^{(0)}\right\rangle$ satisfying (25) and (22) with given $p$ as follow 6

$$
\begin{aligned}
|\Phi\rangle & =\sum_{n=0}^{[p / 2]} \frac{\left(-i q_{1}^{+} p_{1}^{+}\right)^{n}}{n !}\left|\varphi_{p-2 n}\right\rangle+\eta_{0} \sum_{n=1}^{[(p+1) / 2]}\left(q_{1}^{+}\right)^{n-1} \frac{\left(-i p_{1}^{+}\right)^{n}}{n !}\left|\varphi_{p-2 n+1}\right\rangle, \\
\left|\Lambda_{0}^{(0)}\right\rangle & =\sum_{n=1}^{[(p+1) / 2]}\left(q_{1}^{+}\right)^{n-1} \frac{\left(-i p_{1}^{+}\right)^{n}}{n !}\left|\lambda_{p-2 n+1}\right\rangle,
\end{aligned}
$$

where we denote $\left|\varphi_{n}\right\rangle=\frac{(-i)^{n}}{n !} \varphi(x)_{\mu_{1} \ldots \mu_{n}} a^{+\mu_{1}} \ldots a^{+\mu_{n}}|0\rangle$ and $\left|\lambda_{n}\right\rangle=\frac{(-i)^{n}}{n !} \lambda(x)_{\mu_{1} \ldots \mu_{n}} a^{+\mu_{1}} \ldots a^{+\mu_{n}}|0\rangle$. Equation of motion $Q|\Phi\rangle=0$ and gauge transformation $\delta|\Phi\rangle=Q\left|\Lambda_{0}^{(0)}\right\rangle$ looks like

$$
\begin{aligned}
& l_{0}\left|\varphi_{p-2 n}\right\rangle=l_{1}^{+}\left|\varphi_{p-2 n-1}\right\rangle+l_{1}\left|\varphi_{p-2 n+1}\right\rangle, \\
& \left|\varphi_{p-2 n-1}\right\rangle+l_{1}\left|\varphi_{p-2 n}\right\rangle+l_{1}^{+}\left|\varphi_{p-2 n-2}\right\rangle=0, \\
& \delta\left|\varphi_{p-2 n}\right\rangle=l_{1}\left|\lambda_{p-2 n+1}\right\rangle+l_{1}^{+}\left|\lambda_{p-2 n-1}\right\rangle, \\
& \delta\left|\varphi_{p-2 n-1}\right\rangle=l_{0}\left|\lambda_{p-2 n-1}\right\rangle .
\end{aligned}
$$

Using gauge transformation (32) starting with the field with the lowest rank we eliminate all the fields $\left|\varphi_{p-2 n}\right\rangle$ except $\left|\varphi_{p}\right\rangle$. After this all the fields $\left|\varphi_{p-2 n-1}\right\rangle$ except $\left|\varphi_{p-1}\right\rangle$ become zero as consequences equations of motion (31). The rest equations of motion on fields $\left|\varphi_{p}\right\rangle$ and $\left|\varphi_{p-1}\right\rangle$ and the residual gauge transformation with restricted gauge parameter $\left|\lambda_{p-1}\right\rangle$ are

$$
\begin{array}{ll}
l_{0}\left|\varphi_{p}\right\rangle=l_{1}^{+}\left|\varphi_{p-1}\right\rangle, & \delta\left|\varphi_{p}\right\rangle=l_{1}^{+}\left|\lambda_{p-1}\right\rangle, \\
l_{1}\left|\varphi_{p}\right\rangle+\left|\varphi_{p-1}\right\rangle=0, & \delta\left|\varphi_{p-1}\right\rangle=l_{0}\left|\lambda_{p-1}\right\rangle,
\end{array}
$$

Acting on equation of motion (35) by operator $l_{1}$ and using that $l_{1}^{2}=0$ (13) one finds that $l_{1}\left|\varphi_{p-1}\right\rangle=0$. Therefore using the residual gauge transformation with restricted gauge parameter $\left|\lambda_{p-1}\right\rangle$ we can make solution $\left|\varphi_{p-1}\right\rangle$ to be zero $\left|\varphi_{p-1}\right\rangle=0$. As a result only physical field $\left|\varphi_{p}\right\rangle$ is nonvanishing and equations of motion for it are

$$
l_{0}\left|\varphi_{p}\right\rangle=0, \quad l_{1}\left|\varphi_{p}\right\rangle=0 .
$$

Thus we have shown that equations of motion following from Lagrangian (23) gives (2), (14) [or equivalently (8)] up to a gauge fixing.

Let us simplify Lagrangian (23) for antisymmetric field with given rank- $p$. Substituting decom-

\footnotetext{
${ }^{6}$ In decomposition (28) the physical field $\varphi(x)_{\mu_{1} \ldots \mu_{p}}$ is contained in $\left|\varphi_{p}\right\rangle$. All other fields $\left|\varphi_{n}\right\rangle$ are auxiliary ones.
} 
position (28) of the field $|\Phi\rangle$ into (23) one gets

$$
\begin{aligned}
\mathcal{L}= & \left\langle\varphi _ { p } \left|\left\{l_{0}\left|\varphi_{p}\right\rangle-l_{1}^{+}\left|\varphi_{p-1}\right\rangle\right\}+\sum_{n=1}^{[p / 2]-1}\left\langle\varphi _ { p - 2 n } \left|\left\{l_{0}\left|\varphi_{p-2 n}\right\rangle-l_{1}\left|\varphi_{p-2 n+1}\right\rangle-l_{1}^{+}\left|\varphi_{p-2 n-1}\right\rangle\right\}\right.\right.\right.\right. \\
& -\sum_{n=0}^{[(p-3) / 2]}\left\langle\varphi _ { p - 2 n - 1 } \left|\left\{\left|\varphi_{p-2 n-1}\right\rangle+l_{1}\left|\varphi_{p-2 n}\right\rangle+l_{1}^{+}\left|\varphi_{p-2 n-2}\right\rangle\right\}\right.\right. \\
& +\left\{\begin{array}{cc}
p \text { even } & \left\langle\varphi _ { 0 } \left|\left\{l_{0}\left|\varphi_{0}\right\rangle-l_{1}\left|\varphi_{1}\right\rangle\right\}-\left\langle\varphi _ { 1 } \left|\left\{\left|\varphi_{1}\right\rangle+l_{1}\left|\varphi_{2}\right\rangle+l_{1}^{+}\left|\varphi_{0}\right\rangle\right\}\right.\right.\right.\right. \\
p \text { odd } & \left\langle\varphi _ { 1 } \left|\left\{l_{0}\left|\varphi_{1}\right\rangle-l_{1}\left|\varphi_{2}\right\rangle-l_{1}^{+}\left|\varphi_{0}\right\rangle\right\}-\left\langle\varphi _ { 0 } \left|\left\{\left|\varphi_{0}\right\rangle+l_{1}\left|\varphi_{1}\right\rangle\right\}\right.\right.\right.\right.
\end{array}\right.
\end{aligned}
$$

Here field $\left|\varphi_{p}\right\rangle$ is a physical one and the rest fields are auxiliary. Our purpose now is to obtain Lagrangian in terms of one physical field $\left|\varphi_{p}\right\rangle$. For this we express fields $\left|\varphi_{p-2 n+1}\right\rangle$ using their equations of motion $\left|\varphi_{p-2 n+1}\right\rangle=-l_{1}\left|\varphi_{p-2 n+2}\right\rangle-l_{1}^{+}\left|\varphi_{p-2 n}\right\rangle$ and substitute them into Lagrangian (37). Taking into account (13) one can show that all other fields except $\left|\varphi_{p}\right\rangle$ disappear and we get

$$
\mathcal{L}=\left\langle\varphi_{p}\left|\left[l_{0}+l_{1}^{+} l_{1}\right]\right| \varphi_{p}\right\rangle=-\left\langle\varphi_{p}\left|l_{1} l_{1}^{+}\right| \varphi_{p}\right\rangle .
$$

In order to obtain this Lagrangian in component form we substitute into it the explicit expressions for $l_{1}, l_{1}^{+}$, and for $\left|\varphi_{p}\right\rangle=\frac{(-i)^{p}}{p !} \varphi(x)_{\mu_{1} \ldots \mu_{n}} a^{+\mu_{1}} \ldots a^{+\mu_{p}}|0\rangle$ and obtain

$$
\mathcal{L}=-\frac{1}{(p+1) !} F_{\mu_{1} \ldots \mu_{p+1}} F^{\mu_{1} \ldots \mu_{p+1}}
$$

where $F_{\mu_{1} \ldots \mu_{p+1}}$ is the strength of antisymmetric field $\varphi_{\mu_{1} \ldots \mu_{p}}$

$$
F_{\mu_{1} \ldots \mu_{p+1}}=\frac{1}{p !}\left[\nabla_{\mu_{1}} \varphi_{\mu_{2} \ldots \mu_{p+1}} \pm(p+1) !-1 \text { permutations }\right] .
$$

Thus we simplified Lagrangian (23) and obtained Lagrangian in terms of one physical field. It is this form of Lagrangian which is commonly used for antisymmetric massless field.

Let us turn to Lagrangian construction for massive antisymmetric fields.

\section{Lagrangian construction for massive fields}

In the massive case we take the mass-shell equation in the form of the mass-shell equation for the massless antisymmetric field (14) deformed by the mass term

$$
\left(\nabla^{2}-m^{2}\right) \varphi_{\mu_{1} \ldots \mu_{p}}+(-1)^{p} p R_{\left[\mu_{1}\right.}^{\alpha} \varphi_{\left.\mu_{2} \ldots \mu_{p}\right] \alpha}-p(p-1) R_{\left[\mu_{1}\right.}^{\alpha \beta}{ }_{\mu_{2}} \varphi_{\left.\mu_{3} \ldots \mu_{p}\right] \alpha \beta}=0 .
$$

Then we denote operator corresponding to equation (41) as $l_{0}^{(m)}$ and define it as $l_{0}^{(m)}=l_{0}-m^{2}$

$$
l_{0}^{(m)}=D^{2}-m^{2}+R_{\mu \nu \alpha \beta} a^{+\mu} a^{\nu} a^{+\alpha} a^{\beta} .
$$

Now anticommutator $\left\{l_{1}, l_{1}^{+}\right\}$is

$$
\left\{l_{1}, l_{1}^{+}\right\}=-l_{0}^{(m)}-m^{2}
$$

and in order to have a set of operators which is invariant under Hermitian conjugation and which form an algebra we add operator $g_{m}=m^{2}$. As a result the algebra of the operators in the massive case is

$$
\begin{array}{ll}
\left\{l_{1}, l_{1}^{+}\right\}=-l_{0}^{(m)}-g_{m}, \quad\left\{l_{1}, l_{1}\right\}=\left\{l_{1}^{+}, l_{1}^{+}\right\}=\left[l_{1}, l_{0}^{(m)}\right]=\left[l_{1}^{+}, l_{0}^{(m)}\right]=0, \\
{\left[g_{m}, l_{1}\right]=\left[g_{m}, l_{1}^{+}\right]=\left[g_{m}, l_{0}\right]=0 .}
\end{array}
$$


In the set of operators we have one operator $g_{m}$ which is not a constraint neither in the bra nor in the ket-vector space. In this case in order to construct Lagrangian within the BRST approach (see e.g. [6]) we need to introduce additional (new) creation and annihilation operators and then construct extended operators $o_{i} \rightarrow O_{i}=o_{i}+o_{i}^{\prime}, o_{i}=\left(l_{0}^{(m)}, l_{1}, l_{1}^{+}, g_{m}\right)$ which must satisfy two conditions: 1$)$ they must form an algebra $\left[O_{i}, O_{j}\right] \sim O_{k} ; 2$ ) the operators which are not constraints must be zero (that is in the case under consideration we must have $G_{m}=g_{m}+g_{m}^{\prime}=0$ ).

For this purpose we introduce one pair fermionic creation and annihilation operators with the standard commutation relations $\left\{f, f^{+}\right\}=1$ and put

$$
l_{0}^{(m) \prime}=0, \quad l_{1}^{\prime+}=m f^{+}, \quad l_{1}^{\prime}=m f, \quad g_{m}^{\prime}=-m^{2} .
$$

One can check that the extended expressions of the operators satisfy the above requirements: the extended expression for the operator $g_{m}$ which is not a constraint is zerd $G_{m}=0$; the operators $L_{0}^{(m)}, L_{1}, L_{1}^{+}$form an algebra

$$
\left\{L_{1}, L_{1}^{+}\right\}=-L_{0}^{(m)}, \quad\left\{L_{1}, L_{1}\right\}=\left\{L_{1}^{+}, L_{1}^{+}\right\}=\left[L_{1}, L_{0}^{(m)}\right]=\left[L_{1}^{+}, L_{0}^{(m)}\right]=0 .
$$

After this one should construct BRST operator

$$
\begin{array}{rlr}
Q_{m}= & \eta_{0} L_{0}^{(m)}+q_{1}^{+} L_{1}+q_{1} L_{1}^{+}+q_{1}^{+} q_{1} \mathcal{P}_{0}, & Q_{m}^{2}=0, \\
& \sigma_{m}=a_{\mu}^{+} a^{\mu}+f^{+} f+i q_{1}^{+} p_{1}-i p_{1}^{+} q_{1}, & {\left[Q_{m}, \sigma_{m}\right]=0}
\end{array}
$$

with the ghosts satisfying relations (17), (19). In the massive case the general state in the Hilbert space looks as follows

$$
|\Phi\rangle=\sum_{k_{i}} \eta_{0}^{k_{1}}\left(q_{1}^{+}\right)^{k_{2}}\left(p_{1}^{+}\right)^{k_{3}}\left(f^{+}\right)^{k_{4}} a^{+\mu_{1}} \ldots a^{+\mu_{k_{0}}} \Phi_{\mu_{1} \ldots \mu_{k}}^{k_{1} k_{2} k_{3} k_{4}}(x)|0\rangle
$$

The sum in (50) is taken over $k_{1}, k_{4}$ running from 0 to 1 and over $k_{2}, k_{3}, k_{0}$ running from 0 to infinity.

Analogously to the massless case to construct Lagrangian for a field with a given spin $p$ we restrict the fields $|\Phi\rangle$ and the gauge parameters $\left|\Lambda^{(i)}\right\rangle$ in the extended Fock space (50) as follows

$$
\sigma_{m}|\Phi\rangle=p|\Phi\rangle, \quad \sigma_{m}\left|\Lambda^{(i)}\right\rangle=p\left|\Lambda^{(i)}\right\rangle,
$$

with operator $\sigma_{m}$ given in (49) If we omit these conditions then the Lagrangian (and the gauge transformations) will contain fields with all spins. One can show (see e.g. [6]) that Lagrangian can be written as

$$
\mathcal{L}=\int d \eta_{0}\left\langle\Phi\left|Q_{m}\right| \Phi\right\rangle
$$

which is invariant under the reducible gauge transformations

$$
\delta|\Phi\rangle=Q_{m}\left|\Lambda^{(0)}\right\rangle, \quad \ldots \quad \delta\left|\Lambda^{(i)}\right\rangle=Q_{m}\left|\Lambda^{(i+1)}\right\rangle, \quad \ldots \quad \delta\left|\Lambda^{(p-2)}\right\rangle=Q_{m}\left|\Lambda^{(p-1)}\right\rangle .
$$

We note here that in the massive case the gauge symmetry are Stückelberg one. The Stückelberg fields and gauge parameters in decomposition (50) are those which contain $f^{+}$, i.e. fields corresponding to $k_{4}=1: \Phi_{\mu_{1} \ldots \mu_{k_{0}}}^{k_{1} k_{2} k_{3} 1}(x)$. The chain of the gauge transformations (53) is finite due to (51) and to the ghost number restriction

$$
g h(|\Phi\rangle)=0, \quad g h\left(\left|\Lambda^{(i)}\right\rangle=-(i+1) .\right.
$$

\footnotetext{
${ }^{7}$ In what follows we forget about operator $G_{m}=0$.
} 
Thus the Lagrangian for the massive bosonic antisymmetric field in an arbitrary curved background is constructed.

Let us show that Lagrangian (52) gives equations of motion which equivalent (21), (41) up to a gauge transformation. Let the rank of the antisymmetric field be equal to $p$. Then we have a reducible gauge theory with $p-1$ number of reducibility stages. Due to (51) and (54) the lowest stage gauge parameter has the form

$$
\left|\Lambda^{(p-1)}\right\rangle=\frac{(-i)^{p}}{p !}\left(p_{1}^{+}\right)^{p} \xi^{(p-1)}(x)|0\rangle .
$$

It can be explicitly checked that we can eliminate the dependence on $f^{+}$in the gauge function $\left|\Lambda^{(p-2)}\right\rangle$ of the $(p-2)$-stage. Then it is possible to check that we can remove dependence of $\left|\Lambda^{(p-3)}\right\rangle$ on $f^{+}$ with the help of the remaining gauge parameters $\left|\Lambda^{(p-2)}\right\rangle$ (which do not depend on $f^{+}$). Then we can proceed further in the same way and in the end remove dependence of the field $|\Phi\rangle$ on $f^{+}$. Thus we have the gauge $f|\Phi\rangle=0$ and all the gauge parameters have been used.

Let us turn to the equations of motion $Q_{m}|\Phi\rangle=0$. Decomposing in power series of $f^{+}$one finds that a part of the equations of motion has the form

$$
m q_{1} f^{+}|\Phi\rangle=0,
$$

which tells us that the field does not depend on $p_{1}^{+}$. This condition together with the gauge $f|\Phi\rangle=0$ give that the field $|\Phi\rangle$ can depend on $a^{+\mu}$ only. That is it remains only the physical field

$$
|\Phi\rangle=\left|\varphi_{p}\right\rangle=\frac{(-i)^{p}}{p !} \varphi(x)_{\mu_{1} \ldots \mu_{p}} a^{+\mu_{1}} \ldots a^{+\mu_{p}}|0\rangle
$$

and the equations of motion for it are

$$
l_{0}^{(m)}\left|\varphi_{p}\right\rangle=0, \quad l_{1}\left|\varphi_{p}\right\rangle=0 .
$$

In component form these are equations (22), (41) which we wanted to reproduce.

Let us simplify Lagrangian (52). For this purpose we decompose field $|\Phi\rangle$ satisfying (51) with a given $p$ and (54) as follow: 8

$$
\begin{aligned}
|\Phi\rangle= & \sum_{n=0}^{[p / 2]} \frac{\left(-i q_{1}^{+} p_{1}^{+}\right)^{n}}{n !}\left|\varphi_{p-2 n}\right\rangle+\eta_{0} \sum_{n=1}^{[(p+1) / 2]}\left(q_{1}^{+}\right)^{n-1} \frac{\left(-i p_{1}^{+}\right)^{n}}{n !}\left|\varphi_{p-2 n+1}\right\rangle \\
& +f^{+} \sum_{n=0}^{[(p-1) / 2]} \frac{\left(-i q_{1}^{+} p_{1}^{+}\right)^{n}}{n !}\left|\psi_{p-2 n-1}\right\rangle+\eta_{0} f^{+} \sum_{n=1}^{[p / 2]}\left(q_{1}^{+}\right)^{n-1} \frac{\left(-i p_{1}^{+}\right)^{n}}{n !}\left|\psi_{p-2 n}\right\rangle
\end{aligned}
$$

where as before we denote

$$
\left|\varphi_{n}\right\rangle=\frac{(-i)^{n}}{n !} \varphi(x)_{\mu_{1} \ldots \mu_{n}} a^{+\mu_{1}} \ldots a^{+\mu_{n}}|0\rangle, \quad\left|\psi_{n}\right\rangle=\frac{(-i)^{n}}{n !} \psi(x)_{\mu_{1} \ldots \mu_{n}} a^{+\mu_{1}} \ldots a^{+\mu_{n}}|0\rangle .
$$

\footnotetext{
${ }^{8}$ Analogously to massless case the physical field $\varphi(x)_{\mu_{1} \ldots \mu_{p}}$ in decomposition (59) is contained in $\left|\varphi_{p}\right\rangle$. The other fields $\left|\varphi_{n}\right\rangle$ are auxiliary and fields $\left|\psi_{n}\right\rangle$ are Stückelberg ones.
} 
Then we substitute (59) into (52) and obtain

$$
\begin{aligned}
& \mathcal{L}=\left\langle\varphi _ { p } \left|\left\{l_{0}^{(m)}\left|\varphi_{p}\right\rangle-l_{1}^{+}\left|\varphi_{p-1}\right\rangle\right\}+\left\langle\psi _ { p - 1 } \left|\left\{l_{0}^{(m)}\left|\psi_{p-1}\right\rangle-m\left|\varphi_{p-1}\right\rangle+l_{1}^{+}\left|\psi_{p-2}\right\rangle\right\}\right.\right.\right.\right. \\
& +\sum_{n=1}^{[p / 2]-1}\left\langle\varphi _ { p - 2 n } \left|\left\{l_{0}^{(m)}\left|\varphi_{p-2 n}\right\rangle-l_{1}\left|\varphi_{p-2 n+1}\right\rangle-l_{1}^{+}\left|\varphi_{p-2 n-1}\right\rangle-m\left|\psi_{p-2 n}\right\rangle\right\}\right.\right. \\
& -\sum_{n=0}^{[(p-3) / 2]}\left\langle\varphi _ { p - 2 n - 1 } \left|\left\{\left|\varphi_{p-2 n-1}\right\rangle+l_{1}\left|\varphi_{p-2 n}\right\rangle+l_{1}^{+}\left|\varphi_{p-2 n-2}\right\rangle+m\left|\psi_{p-2 n-1}\right\rangle\right\}\right.\right. \\
& +\sum_{n=1}^{[(p-3) / 2]}\left\langle\psi _ { p - 2 n - 1 } \left|\left\{l_{0}^{(m)}\left|\psi_{p-2 n-1}\right\rangle-m\left|\varphi_{p-2 n-1}\right\rangle+l_{1}\left|\psi_{p-2 n}\right\rangle+l_{1}^{+}\left|\psi_{p-2 n-2}\right\rangle\right\}\right.\right. \\
& -\sum_{n=1}^{[p / 2]-1}\left\langle\psi _ { p - 2 n } \left|\left\{\left|\psi_{p-2 n}\right\rangle+m\left|\varphi_{p-2 n}\right\rangle-l_{1}\left|\psi_{p-2 n+1}\right\rangle-l_{1}^{+}\left|\psi_{p-2 n-1}\right\rangle\right\}\right.\right. \\
& +\left\{\begin{array}{cc}
p \text { even } & \left\langle\varphi _ { 0 } \left|\left\{l_{0}^{(m)}\left|\varphi_{0}\right\rangle-l_{1}\left|\varphi_{1}\right\rangle-m\left|\psi_{0}\right\rangle\right\}-\left\langle\varphi _ { 1 } \left|\left\{\left|\varphi_{1}\right\rangle+l_{1}\left|\varphi_{2}\right\rangle+l_{1}^{+}\left|\varphi_{0}\right\rangle+m\left|\psi_{1}\right\rangle\right\}\right.\right.\right.\right. \\
p \text { odd } & \left\langle\varphi _ { 1 } \left|\left\{l_{0}^{(m)}\left|\varphi_{1}\right\rangle-l_{1}\left|\varphi_{2}\right\rangle-l_{1}^{+}\left|\varphi_{0}\right\rangle-m\left|\psi_{1}\right\rangle\right\}-\left\langle\varphi _ { 0 } \left|\left\{\left|\varphi_{0}\right\rangle+l_{1}\left|\varphi_{1}\right\rangle+m\left|\psi_{0}\right\rangle\right\}\right.\right.\right.\right.
\end{array}\right. \\
& +\left\{\begin{array}{cl}
p \text { even } & \left\langle\psi _ { 1 } \left|\left\{l_{0}^{(m)}\left|\psi_{1}\right\rangle-m\left|\varphi_{1}\right\rangle+l_{1}\left|\psi_{2}\right\rangle+l_{1}^{+}\left|\psi_{0}\right\rangle\right\}-\left\langle\psi _ { 0 } \left|\left\{\left|\psi_{0}\right\rangle+m\left|\varphi_{0}\right\rangle-l_{1}\left|\psi_{1}\right\rangle\right\}\right.\right.\right.\right. \\
p \text { odd } & \left\langle\psi _ { 0 } \left|\left\{l_{0}^{(m)}\left|\psi_{0}\right\rangle-m\left|\varphi_{0}\right\rangle+l_{1}\left|\psi_{1}\right\rangle\right\}-\left\langle\psi _ { 1 } \left|\left\{\left|\psi_{1}\right\rangle+m\left|\varphi_{1}\right\rangle-l_{1}\left|\psi_{2}\right\rangle-l_{1}^{+}\left|\psi_{0}\right\rangle\right\}\right.\right.\right.\right.
\end{array}\right.
\end{aligned}
$$

After this we substitute equations of motion $\left|\varphi_{p-2 n-1}\right\rangle=-l_{1}\left|\varphi_{p-2 n}\right\rangle-l_{1}^{+}\left|\varphi_{p-2 n-2}\right\rangle-m\left|\psi_{p-2 n-1}\right\rangle$ and $\left|\psi_{p-2 n}\right\rangle=l_{1}\left|\psi_{p-2 n+1}\right\rangle+l_{1}^{+}\left|\psi_{p-2 n-1}\right\rangle-m\left|\varphi_{p-2 n}\right\rangle$ into Lagrangian (61) and find

$$
\begin{aligned}
\mathcal{L} & =\left\langle\varphi _ { p } \left|\left\{\left[l_{0}^{(m)}+l_{1}^{+} l_{1}\right]\left|\varphi_{p}\right\rangle+m l_{1}^{+}\left|\psi_{p-1}\right\rangle\right\}+\left\langle\psi _ { p - 1 } \left|\left\{\left[l_{0}^{(m)}+m^{2}+l_{1}^{+} l_{1}\right]\left|\psi_{p-1}\right\rangle+m l_{1}\left|\varphi_{p}\right\rangle\right\}\right.\right.\right.\right. \\
& =-\left\langle\varphi_{p}\left|\left[l_{1} l_{1}^{+}+m^{2}\right]\right| \varphi_{p}\right\rangle-\left\langle\psi_{p-1}\left|l_{1} l_{1}^{+}\right| \psi_{p-1}\right\rangle+\left\langle\varphi_{p}\left|m l_{1}^{+}\right| \psi_{p-1}\right\rangle+\left\langle\psi_{p-1}\left|m l_{1}\right| \varphi_{p}\right\rangle .
\end{aligned}
$$

In order to get gauge transformations which leave this Lagrangian invariant we decompose gauge parameters $\left|\Lambda^{(i)}\right\rangle$ satisfying (54) and (51) with given $p$ as follows

$$
\begin{aligned}
\left|\Lambda^{(i)}\right\rangle= & \sum_{n=0}^{[(p-i-1) / 2]}\left(q_{1}^{+}\right)^{n} \frac{\left(-i p_{1}^{+}\right)^{n+i+1}}{(n+i+1) !}\left|\lambda_{p-2 n-i-1}^{(i)}\right\rangle+\eta_{0} \sum_{n=0}^{[(p-i) / 2]}\left(q_{1}^{+}\right)^{n} \frac{\left(-i p_{1}^{+}\right)^{n+i+2}}{(n+i+2) !}\left|\lambda_{p-2 n-i}^{(i)}\right\rangle \\
& +f^{+} \sum_{n=0}^{[(p-i-2) / 2]}\left(q_{1}^{+}\right)^{n} \frac{\left(-i p_{1}^{+}\right)^{n+i+1}}{(n+i+1) !}\left|\lambda_{p-2 n-i-2}^{(i)}\right\rangle+\eta_{0} f^{+} \sum_{n=0}^{[(p-i-1) / 2]}\left(q_{1}^{+}\right)^{n} \frac{\left(-i p_{1}^{+}\right)^{n+i+2}}{(n+i+2) !}\left|\lambda_{p-2 n-i-1}^{(i)}\right\rangle
\end{aligned}
$$

Substituting this decomposition into (53) one finds the chain of reducible gauge transformation under which (62) is invariant

$$
\begin{array}{ll}
\delta\left|\varphi_{p}\right\rangle=l_{1}^{+}\left|\lambda_{p-1}\right\rangle, & \delta\left|\psi_{p-1}\right\rangle=m\left|\lambda_{p-1}\right\rangle+l_{1}^{+}\left|\omega_{p-2}\right\rangle \\
\ldots & \ldots \\
\delta\left|\lambda_{p-i-1}^{(i)}\right\rangle=l_{1}^{+}\left|\lambda_{p-i-2}^{(i+1)}\right\rangle, & \delta\left|\omega_{p-i-2}^{(i)}\right\rangle=m\left|\lambda_{p-i-2}^{(i+1)}\right\rangle+l_{1}^{+}\left|\omega_{p-i-3}^{(i+1)}\right\rangle, \\
\ldots & \cdots \\
\delta\left|\lambda_{1}^{(p-2)}\right\rangle=l_{1}^{+}\left|\lambda_{0}^{(p-1)}\right\rangle, & \delta\left|\omega_{0}^{(p-2)}\right\rangle=m\left|\lambda_{0}^{(p-1)}\right\rangle .
\end{array}
$$

We see that field $\left|\psi_{p-1}\right\rangle$ and gauge parameters $\left|\omega_{p-i-2}^{(i)}\right\rangle$ are responsible for the presence of the Stückelberg symmetry. We can partially fix the gauge symmetry and get rid of the Stückelberg gauge 
parameters $\left|\omega_{p-i-2}^{(i)}\right\rangle$. After this Lagrangian (하) will be invariant under the following Stückelberg symmetry transformation

$$
\delta\left|\varphi_{p}\right\rangle=l_{1}^{+}\left|\lambda_{p-1}\right\rangle, \quad \delta\left|\psi_{p-1}\right\rangle=m\left|\lambda_{p-1}\right\rangle .
$$

Let us write Lagrangian (62) and gauge transformations (67) in component form. One has

$$
\begin{gathered}
\mathcal{L}=-\frac{1}{(p+1) !} F_{\mu_{1} \ldots \mu_{p+1}} F^{\mu_{1} \ldots \mu_{p+1}}-\frac{m^{2}}{p !} \varphi_{\mu_{1} \ldots \mu_{p}} \varphi^{\mu_{1} \ldots \mu_{p}} \\
-\frac{1}{p !} f_{\mu_{1} \ldots \mu_{p}} f^{\mu_{1} \ldots \mu_{p}}+\frac{2 m}{p !} \varphi_{\mu_{1} \ldots \mu_{p}} f^{\mu_{1} \ldots \mu_{p}} \\
\delta \varphi_{\mu_{1} \ldots \mu_{p}}=p \nabla_{\left[\mu_{1}\right.} \lambda_{\left.\mu_{2} \ldots \mu_{p}\right]}, \quad \delta \psi_{\mu_{1} \ldots \mu_{p-1}}=m \lambda_{\mu_{1} \ldots \mu_{p-1}}
\end{gathered}
$$

where $F_{\mu_{1} \ldots \mu_{p+1}}$ is the strength (40) of antisymmetric field $\varphi_{\mu_{1} \ldots \mu_{p}}$ and $f_{\mu_{1} \ldots \mu_{p}}$ is the strength of field $\psi_{\mu_{1} \ldots \mu_{p-1}}$. One can proceed further and fix gauge completely removing field $\psi_{\mu_{1} \ldots \mu_{p-1}}$. Finally we obtain Lagrangian

$$
\mathcal{L}=-\frac{1}{(p+1) !} F_{\mu_{1} \ldots \mu_{p+1}} F^{\mu_{1} \ldots \mu_{p+1}}-\frac{m^{2}}{p !} \varphi_{\mu_{1} \ldots \mu_{p}} \varphi^{\mu_{1} \ldots \mu_{p}}
$$

which has no symmetry transformations. Thus we simplified Lagrangian (52) and obtained Lagrangian (70) which is commonly used for antisymmetric massive bosonic field.

\section{Summary}

We have shown that the BRST approach, which was developed earlier for higher spin field models in flat and AdS spaces, perfectly works for massless and massive bosonic antisymmetric fields in arbitrary curved space-time. The obtained theories are reducible gauge models, the corresponding Lagrangians and gauge transformations are given by (23), (24) and (152), (153) respectively for massless and massive theories. In both the theories the order of reducibility grows with the value of the rank of the antisymmetric field 9 . In the massive case we automatically get a formulation with appropriate Stückelberg fields. Like all the Lagrangians constructed on the base of the BRST approach, the obtained Lagrangians possess more rich gauge symmetry and contain more fields in comparison with those which are commonly used for description of the antisymmetric fields. Due to the presence of the additional symmetry it is possible to get various intermediate Lagrangian formulations (like (68)) for massive and massless antisymmetric field theories by partial gauge fixing and eliminating some of the auxiliary fields.

\section{Acknowledgements}

The work of I.L.B, V.A.K and L.L.R. was partially supported by RF Presidential grant for LSS, project No. 4489.2006.2. The work of I.L.B and V.A.K was partially supported by the INTAS grant, project INTAS-05-7928 and the RFBR grant, project No. 06-02-16346. The work of I.L.B was also supported in part by the DFG grant, project No. 436 RUS 113/669/0-3 and joint RFBR-DFG grant, project No. 06-02-04012.

\footnotetext{
${ }^{9}$ In massless case this statement obviously corresponds to structure of gauge transformations in conventional formulation. However, in massive case, the approach under consideration also leads to reducible gauge models.
} 


\section{References}

[1] C. Becchi, A. Rouet and R. Stora, Renormalization Of The Abelian Higgs-Kibble Model, Commun. Math. Phys. 42 (1975) 127; Renormalization Of Gauge Theories, Annals Phys. 98 (1976) 287; I.V. Tyutin, Gauge invariance in field theory and statistics in operator formulation, preprint FIAN, No. 39 (1975); E.S. Fradkin, G.A. Vilkovisky, Quantization of relativistic systems with constraints, Phys.Lett. B55 (1975) 224; I.A. Batalin, G.A. Vilkovisky, Relativistic S-matrix of dynamical systems with boson and fermion constraints, Phys.Lett. B69 (1977) 309; I.A. Batalin, E.S. Fradkin, Operator qunatization of relativistic dynamical system subject to first class constraints, Phys.Lett. B128 (1983) 303; I.A. Batalin, Operator quantization method and abelization of dynamical systems subject to first class constraints, Riv.Nuovo.Cim., 9 (1986) No. 10, 1; I.A. Batalin, E.S. Fradkin, Operatorial quantization of dynamical systems subject to constraints. A further study of the construction, Annals Inst. H. Poincare, Theor.Phys. 49 (1988) No2, 145.

[2] M. Vasiliev, Higher Spin Gauge Theories in Various Dimensions, Fortsch.Phys. 52 (20040, 702717; D. Sorokin, Introduction to Classical Theory of Higher Spins, AIP, Conf.Proc. 767 (2005) 172-202, hep-th/0405069; N. Bouatta, G. Compere and A. Sagnotti, An Introduction to Free Higher-Spin Fields, hep-th/0409068; X. Bekaert, S. Cnockaert, C. Iazeola, M. A. Vasiliev, Nonlinear higher spin theories in various dimensions, hep-th/0503128.

[3] A. Pashnev and M. Tsulaia, "Description of the higher massless irreducible integer spins in the BRST approach," Mod. Phys. Lett. A 13 (1998) 1853 [arXiv:hep-th/9803207]. I. L. Buchbinder, A. Pashnev and M. Tsulaia, "Lagrangian formulation of the massless higher integer spin fields in the AdS background," Phys. Lett. B 523 (2001) 338 [arXiv:hep-th/0109067]; I. L. Buchbinder, A. Pashnev and M. Tsulaia, "Massless higher spin fields in the AdS background and BRST constructions for nonlinear algebras," arXiv:hep-th/0206026; X. Bekaert, I. L. Buchbinder, A. Pashnev and M. Tsulaia, "On higher spin theory: Strings, BRST, dimensional reductions," Class. Quant. Grav. 21 (2004) S1457 arXiv:hep-th/0312252]; A. Fotopoulos, K. L. Panigrahi and M. Tsulaia, "Lagrangian Formulation Of Higher Spin Theories On Ads Space," Phys. Rev. D 74 (2006) 085029 arXiv:hep-th/0607248.

[4] I. L. Buchbinder, A. V. Galajinsky and V. A. Krykhtin, "Quartet unconstrained formulation for massless higher spin fields," Nucl. Phys. B 779 (2007) 155-177 arXiv:hep-th/0702161].

[5] I. L. Buchbinder, V. A. Krykhtin and A. Pashnev, "BRST approach to Lagrangian construction for fermionic massless higher spin fields," Nucl. Phys. B 711 (2005) 367 arXiv:hep-th/0410215.

[6] I. L. Buchbinder and V. A. Krykhtin, "Gauge invariant Lagrangian construction for massive bosonic higher spin fields in D dimensions," Nucl. Phys. B 727 (2005) 537 arXiv:hep-th/0505092. I. L. Buchbinder, V. A. Krykhtin and P. M. Lavrov, Nucl. Phys. B 762 (2007) 344-376 |arXiv:hep-th/0608005|. I. L. Buchbinder and V. A. Krykhtin, "BRST approach to higher spin field theories," arXiv:hep-th/0511276; I. L. Buchbinder and V. A. Krykhtin, "Progress in Gauge Invariant Lagrangian Construction for Massive Higher Spin Fields," arXiv:0710.5715 [hep-th].

[7] I. L. Buchbinder, V. A. Krykhtin, L. L. Ryskina and H. Takata, "Gauge invariant Lagrangian construction for massive higher spin fermionic fields," Phys. Lett. B 641 (2006) 386 arXiv:hep-th/0603212 ; I. L. Buchbinder, V. A. Krykhtin and A. A. Reshetnyak, "BRST approach to Lagrangian construction for fermionic higher spin fields in AdS space," Nucl. Phys. B 787 (2007) 211 arXiv:hep-th/0703049. 
[8] C. Burdik, A. Pashnev and M. Tsulaia, "On the mixed symmetry irreducible representations of the Poincare group in the BRST approach," Mod. Phys. Lett. A 16 (2001) 731 arXiv:hep-th/0101201]; P. Y. Moshin and A. A. Reshetnyak, "BRST approach to Lagrangian formulation for mixed-symmetry fermionic higher-spin fields," JHEP 0710 (2007) 040 arXiv:0707.0386 [hep-th]]; I. L. Buchbinder, V. A. Krykhtin and H. Takata, "Gauge invariant Lagrangian construction for massive bosonic mixed symmetry higher spin fields," Phys. Lett. B 656 (2007) 253 [arXiv:0707.2181 [hep-th]].

[9] I. L. Buchbinder, A. Fotopoulos, A. C. Petkou and M. Tsulaia, "Constructing the cubic interaction vertex of higher spin gauge fields," Phys. Rev. D 74 (2006) 105018 [arXiv:hep-th/0609082]; A. Fotopoulos and M. Tsulaia, "Interacting Higher Spins and the High Energy Limit of the Bosonic String," Phys. Rev. D 76 (2007) 025014 [arXiv:0705.2939 [hep-th]]; A. Fotopoulos, N. Irges, A. C. Petkou and M. Tsulaia, "Higher-Spin Gauge Fields Interacting with Scalars: The Lagrangian Cubic Vertex," JHEP 0710 (2007) 021 [arXiv:0708.1399 [hep-th]]; A. Fotopoulos and M. Tsulaia, "Gauge Invariant Lagrangians for Free and Interacting Higher Spin Fields. A Review of the BRST formulation," arXiv:0805.1346 [hep-th].

[10] L. Brink, R.R. Metsaev, M.A. Vasiliev, "How massless are massless fields in $A d S_{d}$," Nucl. Phys. B586 (2000) 183-205, arXiv:hep-th/0005136.

[11] Yu. M. Zinoviev, "On massive mixed symmetry tensor fields in Minkowski space and (A)dS," arXiv:hep-th/0211233; "First order formalism for mixed symmetry tensor fields," arXiv:hep-th/0304067; "First order formalism for massive mixed symmetry tensor fields in Minkowski and (A)dS spaces," arXiv:hep-th/0306292; "On dual formulations of massive tensor fields," JHEP 0510 (2005) 075 [arXiv:hep-th/0504081].

[12] R. R. Metsaev, "Mixed symmetry massive fields in AdS(5)," Class. Quant. Grav. 22 (2005) 2777-2796 arXiv:hep-th/0412311]; "Massless arbitrary spin fields in AdS(5)," Phys. Lett. B 531 (2002) 152-160 |arXiv:hep-th/0201226|; "Arbitrary spin massless bosonic fields in d-dimensional anti-de Sitter space," arXiv:hep-th/9810231; "Fermionic fields in the d-dimensional anti-de Sitter spacetime," Phys. Lett. B 419 (1998) 49-56 [arXiv:hep-th/9802097]; "Massless mixed symmetry bosonic free fields in d-dimensional anti-de Sitter space-time," Phys. Lett. B 354 (1995) 78-84.

[13] R. R. Metsaev, "Free totally (anti)symmetric massless fermionic fields in d-dimensional antide Sitter space," Class. Quant. Grav. 14 (1997) L115-L121 arXiv:hep-th/9707066]; "Lowest eigenvalues of the energy operator for totally (anti)symmetric massless fields of the n-dimensional anti-de Sitter group," Class. Quant. Grav. 11 (1994) L141-L145.

[14] K. B. Alkalaev, O. V. Shaynkman and M. A. Vasiliev, "On the frame-like formulation of mixedsymmetry massless fields in (A)dS(d)," Nucl. Phys. B 692 (2004) 363 arXiv:hep-th/0311164; "Lagrangian formulation for free mixed-symmetry bosonic gauge fields in (A)dS(d)," JHEP 0508 (2005) 069 arXiv:hep-th/0501108; "Frame-like formulation for free mixed-symmetry bosonic massless higher-spin fields in AdS(d)," arXiv:hep-th/0601225.

[15] E.D. Skvortsov, "Mixed-Symmetry Massless Fields in Minkowski space Unfolded," JHEP 0807 (2008) 004 [arXiv:0801.2268 [hep-th]]; "Frame-like Actions for Massless Mixed-Symmetry Fields in Minkowski space," arXiv:0807.0903 [hep-th]. 